\title{
THE MAGNETIC FIELD OF M 83
}

\author{
N. NEININGER ${ }^{1}$, U. KLEIN ${ }^{2}$, R. BECK ${ }^{1}$ and R. WIELEBINSKI ${ }^{1}$ \\ ${ }^{1}$ Max-Planck-Institut für Radioastronomie, Auf dem Hügel 69, W-5300 Bonn 1, FRG \\ ${ }^{2}$ Radioastronomisches Institut der Universität, Auf dem Hügel 71, W-5300 Bonn 1, FRG
}

This southern galaxy with its fairly regular pattern is well suited to study the influence of a bar onto the magnetic field in a galaxy. With its low inclination of $24^{\circ}$ it provides a geometry similar to M 51. Also the magnetic field strength of $11 \pm 2 \mu \mathrm{G}$ is in the same range.

M 83 shows a rather regular distribution of the polarized emission but with remarkable differences between $\lambda 2.8 \mathrm{~cm}$ (Fig. 1a) and $\lambda 20.5 \mathrm{~cm}$ (Fig. 1b). At the longer wavelength the Faraday depolarization is so strong that the galactic disk itself is invisible; only two giant arcs of emission remain at the border of the optically visible disk.

The highest polarized intensity at $\lambda 2.8 \mathrm{~cm}$ is seen in the center and on the bar of this galaxy with severe depressions at the inside of the bar/spiral arm corner. These depressions are accompanied by steep gradients in the rotation measures (RM). The observed field strength cannot produce these RM's if the field is assumed to lie in the plane of the galaxy. Only a field component perpendicular to the plane with a strength of a few $\mu \mathrm{G}$ is able to explain these observations.

The distribution of the RM in the plane of the galaxy shows similarities to the general structure (Fig. 2); the orientation and symmetry axes of the pattern are connected with the bar. An analysis shows that this bisymmetric structure does not behave in the way predicted by the $\alpha \omega$-dynamo in a BSS mode.

In the outskirts of this galaxy the degree of polarization rises up to $60 \%$ which must be due to a well-ordered field in these more quiescent regions.

The $\lambda 2.8 \mathrm{~cm}$ map represents the orientation of the magnetic field within $1^{\circ}$ although the RM values are locally rather high. It shows a regular magnetic field pattern which is mostly aligned with the optical structure; even in the central region the field is oriented along the bar. This is not expected if an $\alpha \omega$-dynamo generates the magnetic field. The depressions seen at $\lambda 2.8 \mathrm{~cm}$ originate from the abrupt change of the magnetic field orientation within the beam size at the bar/spiral arm transition.

The close correspondence of the magnetic field orientation and the optical structure all over the galaxy also favours an amplification mechanism that is directly connected to the dynamics of the galaxy instead of the $\alpha \omega$-dynamo (which is only influenced by the axisymmetric structure of a galaxy). This can be maintained by an amplification process originating from non-axisymmetric disturbances of the galactic disk (see, e.g., Lesch 1993).

\section{References}

Lesch, H.: 1993 (this volume)

Neininger, N., Klein, U., Beck, R., Wielebinski, R.: 1991, Nature 352, 781

Sukumar, S., Allen, R.J.: 1989, Nature 340, 537 

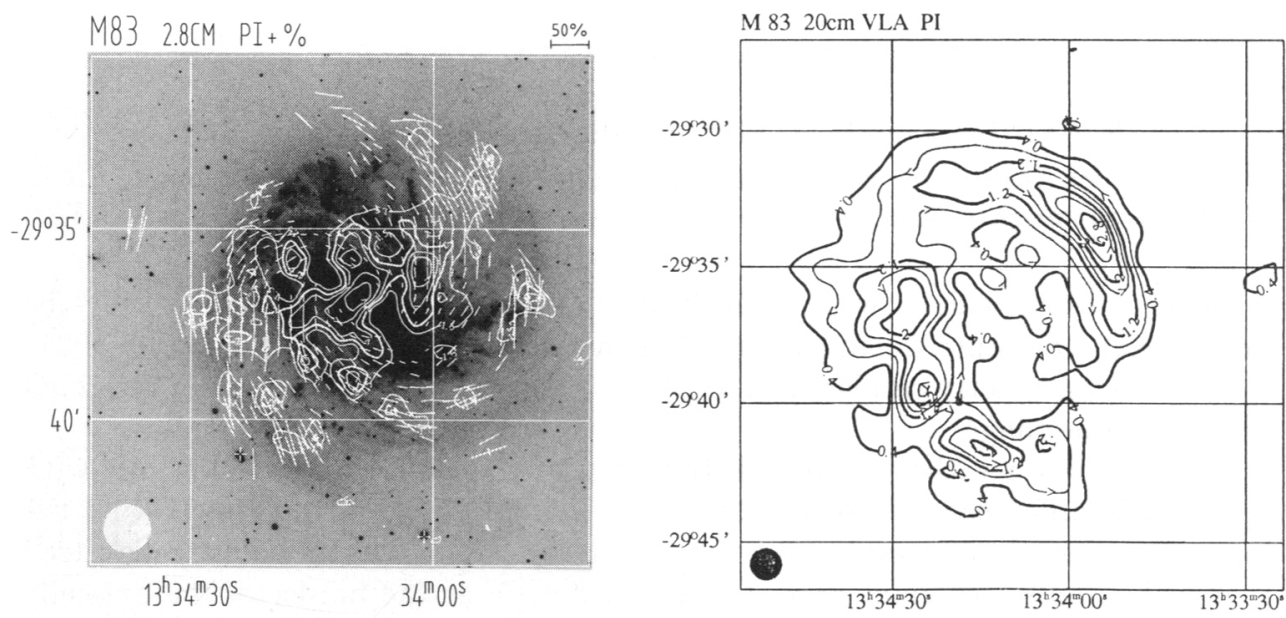

Fig. 1. Polarized Emission of $\mathrm{M} 83$ :

a) Polarized Intensity at $\lambda 2.8 \mathrm{~cm}$ with vectors indicating the B-field orientation (resolution $75^{\prime \prime}$ ) superimposed onto an optical photograph by D. Malin. The vector length is proportional to the degree of polarization.

b) Polarized Intensity at $\lambda 20 \mathrm{~cm}$ (taken from Sukumar and Allen (1989)) smoothed to $75^{\prime \prime}$ resolution. The grid is the same as in (a), but the scale is different: note the large extent of the emission.

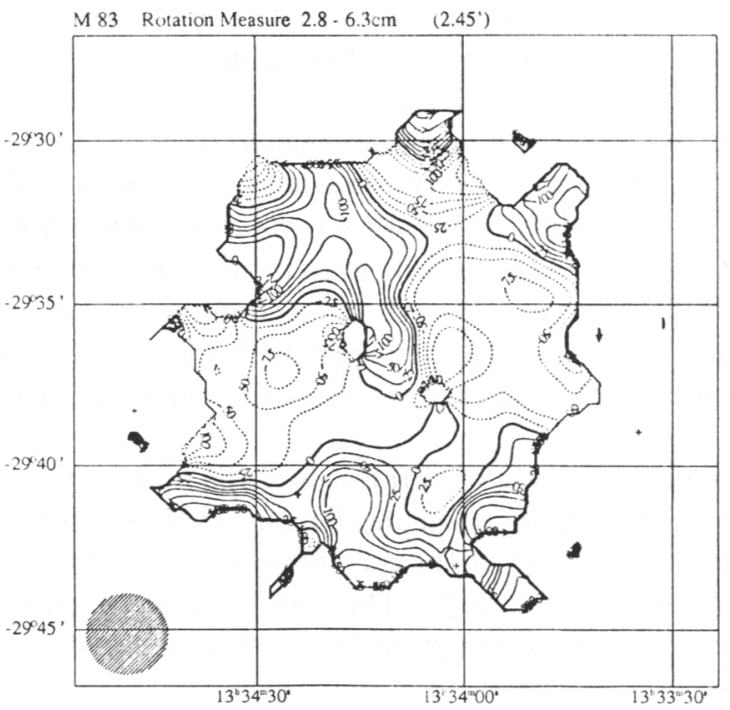

Fig. 2. Map of the rotation measures as determined between $\lambda 2.8 \mathrm{~cm}$ and $\lambda 6.3 \mathrm{~cm}$ with a resolution of $2.45^{\prime}$. Note the double-periodical shape and the steep gradients located besides the bar. However, a fit to the $\mathrm{RM}$ distribution does not indicate a BSS since the phase of adjacent rings does not change as required. Due to the discrepancies in the spatial distribution of the emission and the Faraday depth it is not possible to obtain a well established RM map between $\lambda 2.8 \mathrm{~cm}$ and $\lambda 20 \mathrm{~cm}$. 